\title{
Effects of Intra-ovarian Bursal Injection of Eicosanoid Synthesis Inhibitors on Luteal Function in Immature Pseudopregnant Rats
}

\author{
Shiro KURUSU, Yumi HIRANO, Saori KAWAGISHI, \\ Tsuzumi KITABATAKE, Akinori SUZUKI, \\ Mitsumori KAWAMINAMI and Inoru HASHIMOTO
}

Laboratory of Veterinary Physiology, Kitasato University

School of Veterinary Medicine and Animal Sciences,

Towada, Aomori 034, Japan

\begin{abstract}
To investigate the role of ovarian endogenous eicosanoids in regulation of luteal function, we examined effects of local and chronic administration of eicosanoid synthesis inhibitors on plasma progesterone levels in immature pseudopregnant rats. Injections of drugs in $20 \mu 1$ oil were performed into the ovarian bursal cavity and repeated three times every $36 \mathrm{~h}$. As compared with the control (oil-injected) group,the phospholipase $A_{2}$ inhibitor dexamethasone (DEX) at the dose of $62.5 \mu \mathrm{g} /$ ovary delayed functional luteolysis for 3 days by treatments from day 6 of pseudopregnancy (D6). Indomethacin (IND) also extended luteal activity for 5 days after treatment from D6, while nordihydroguaiaretic acid (NDGA) extended it for only 1 day. The extension of pseudopregnancy by each drug was not so evident with treatments from D2 or D4 as compared to that from D6. From D6, imidazole also delayed luteolysis, while a doubled dose of NDGA did not. Systemic administration of DEX or IND increased plasma progesterone levels more rapidly and significantly than local treatments but were less effective in delaying luteolysis. These results suggest that ovarian endogenous eicosanoids, mainly prostanoids, in the late luteal phase are involved in functional luteolysis in the present model.
\end{abstract}

Key words: Luteolysis, Dexamethasone, Indomethacin, Nordihydroguaiaretic acid, Rat.

(J. Reprod. Dev. 41: 21-28, 1995)

E icosanoids exert a variety of biological activities including regulation of mammalian luteal function. In rats, there are two possible sources of eicosanoids relevant to luteal function; one is the uterus and the other is the corpus luteum itself [1, 2]. Previous studies show that in vivo levels of several prostanoids in the luteal tissue increased or maintained high levels during luteal regression in pseudopregnant rats [3-6], suggesting a role of luteal endogenous eicosanoids in luteolysis. However, one of those studies demonstrated a

Accepted for publication: August 24, 1994 Correspondence: S. Kurusu concomitant increase in prostaglandin $\mathrm{F}_{2 \alpha}\left(\mathrm{PGF}_{2 \alpha}\right)$ in the uterus [5]. The relative importance of $\mathrm{PGF}_{2 \alpha}$ originating from these tissues in regulation of luteal function and lifespan has not been well clarified.

The effects of systemic administration of eicosanoid synthesis inhibitors on rat luteal function were investigated previously $[7,8]$. Furthermore, the effect of intraluteal infusion of a prostaglandin synthesis inhibitor on the luteal function and lifespan was tested using rhesus monkeys [9]; however, such an examination has not been done in rats. In the present study, we examined effects of eicosanoid synthesis inhibitors on plas- 
ma levels of progesterone in immature pseudopregnant rats by local administration of four kinds of inhibitors.

\section{Materials and Methods}

\section{Reagents and animals}

PMSG was purchased from Shionogi Co. (Osaka, Japan). Ovine prolactin and inhibitors of eicosanoid biosynthesis, dexamethasone (DEX), indomethacin (IND), imidazole (IMZ) and nordihydroguaiaretic acid (NDGA) were from Sigma (St. Louis, MO, USA). Immature female Wistar rats were kept in an air-conditioned room with controlled light regimens (lights on $0500 \mathrm{~h}-1900 \mathrm{~h}$ ). Immature, 26 day-old rats were given 5 IU PMSG to induce ovulation and pseudopregnancy. By vaginal smear examinations, the animals exhibited proestrus on day 28; estrus and ovulation occurred on the 29th day. Thereafter, diestrus continued for $13.5 \pm 0.5$ (mean \pm SEM, $n=12$ ) days. Therefore, the 30th day in age was defined as D1 (day 1 of pseudopregnancy) in this study. To ensure a functional corpus luteum, animals in the present model were given subcutaneously 10 IU ovine prolactin twice daily from D1 to D5.

\section{Experimental procedures}

Methods for local administration of drugs were as follows. Bilateral abdominal incisions were made under ether anesthesia. The ovarian bursa was exposed and ligated with thread across the oviduct but not across the blood vessels. Injections of drugs dissolved in sesame oil ( $20 \mu \mathrm{l}$ per side) were performed into the ovarian bursal cavity using a microliter syringe. This treatment started at $0700 \mathrm{~h}$ on D2, D4 or D6 and was repeated three times at $36 \mathrm{~h}$ intervals. During and after the second injections, any remaining vehicle was removed before applying a new one. As we confirmed that the control (oil injection) animal showed a similar progesterone secretion pattern and luteal lifespan as intact animals, the present experimental procedures, such as repeated abdominal operations and oviductal ligation, had no serious effects on luteal activity. Eicosanoid synthesis inhibitors were prepared at the standard concentrations of $62.5 \mu \mathrm{g} /$ ovary or $125 \mu \mathrm{g} /$ rat, which was chosen with reference to previous reports using systemically administered DEX or IND in immature rats $[8,10]$.
As the drugs were most effective in extending luteal function with treatment from D6, experiments of systemic (subcutaneous) treatment with DEX or IND at the same dose were undertaken from D6. Furthermore, experiments of local treatment with variable doses of drugs (indicated in the Results) were also undertaken on D6.

\section{Progesterone assay and statistics}

Systemic blood (about $200 \mu \mathrm{l}$ ) was collected from the tail vein at noon every other day. Mean plasma progesterone levels were determined daily in one of the duplicate experimental groups for each treatment; that is, responses were documented on days $2,4,6,8,10,12$ and 14 in one group and in another group on days $3,5,7,9,11,13$. Plasma progesterone levels were determined by radioimmunoassay as described earlier [11]. The interand intra-assay coefficients of variances were 6$8 \%$ and $12-14 \%$, respectively. All data were displayed as mean \pm SEM $(n=6)$. The means of different treatment groups from the same collection time were analysed by one-way ANOVA and least significant difference test. The level of significance was established to be $\mathrm{P}<0.05$.

\section{Results}

\section{Effects of DEX on progesterone secretion}

The effects of DEX, a phospholipase $\mathrm{A}_{2}$ inhibitor, on luteal activity of immature pseudopregnant rats are shown in Fig. 1. Local injection of DEX $(62.5 \mu \mathrm{g} /$ ovary, $125 \mu \mathrm{g} / \mathrm{rat})$ from D2 induced no effect on plasma progesterone levels (Fig. 1-A). However, treatment from D4 tended to increase plasma progesterone as compared to the control group (Fig. 1-B). When the onset of treatment was delayed to D6, prolonged secretion of progesterone was evident, and half of that dose of DEX was sufficient to induce prolonged luteal function (Fig. 1-C). After systemic treatment with DEX (125 $\mu \mathrm{g} /$ rat) from D6, plasma progesterone levels became significantly higher on D6 and D7 than those of control or locally injected groups and tended to decrease to the basal level earlier (Fig. 1-D).

\section{Effects of IND on progesterone secretion}

When the rats were locally treated with a cyclooxygenase inhibitor IND (62.5 $\mu \mathrm{g} /$ ovary) from D2, they showed a similar pattern in progesterone 

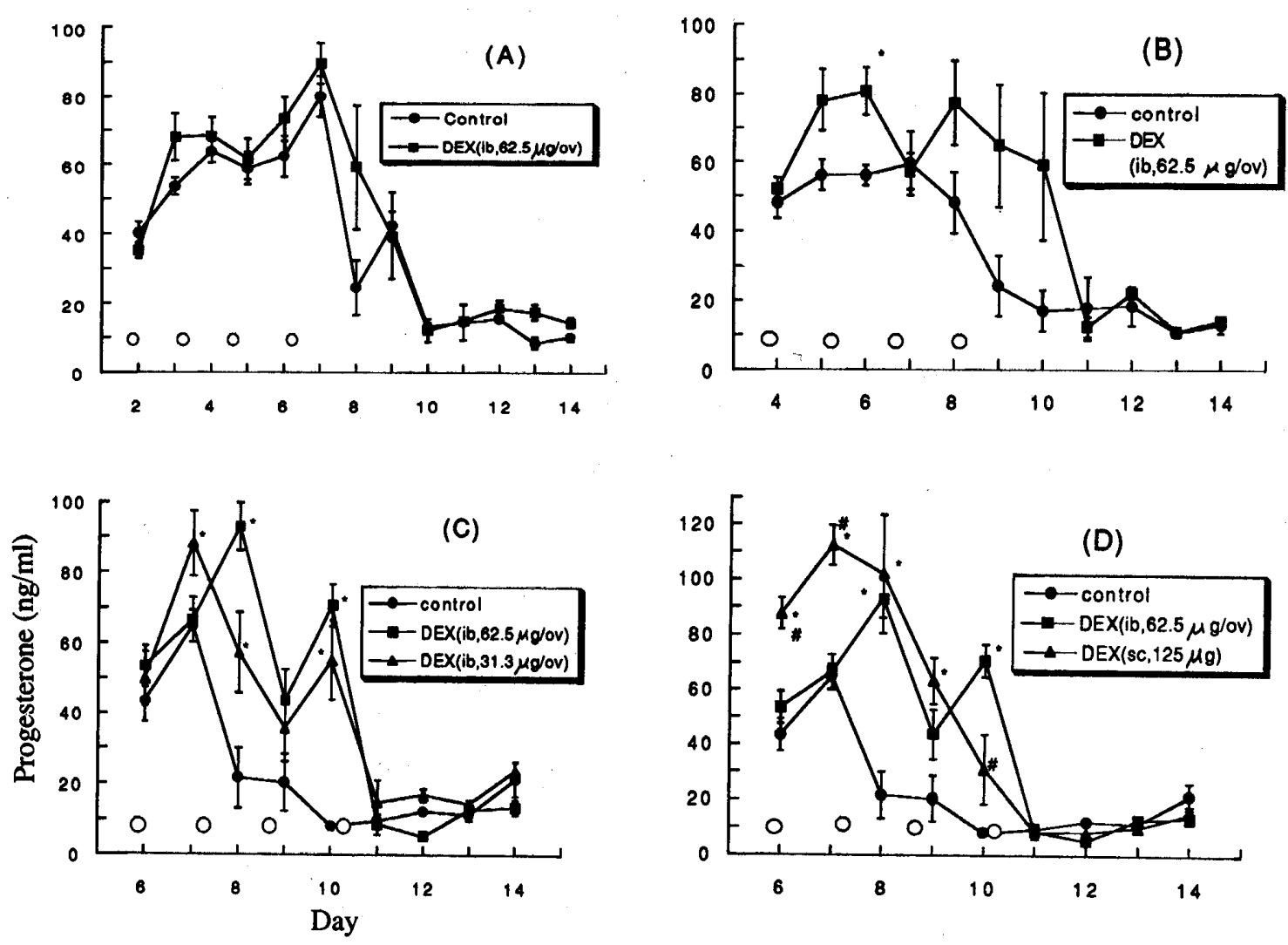

Fig. 1. Changes in plasma progesterone levels in immature pseudopregnant rats following intra-ovarian bursal (ib) or subcutaneous (sc) injections of DEX. The ordinate and abscissa show plasma progesterone levels $(\mathrm{ng} / \mathrm{ml})$ and day of pseudopregnancy, respectively. The injections of drug or vehicle are shown by the open circle $(\bigcirc)$ in each experiment. The doses of DEX in a single injection were $62.5 \mu \mathrm{g} /$ ovary $(125 \mu \mathrm{g} / \mathrm{rat})$ and $31.25 \mu \mathrm{g} /$ ovary $(62.5 \mu \mathrm{g} / \mathrm{rat})$ in ib injected groups and $125 \mu \mathrm{g} / \mathrm{rat}$ in sc injected groups. *: significantly different from the control group $(\mathrm{P}<0.05)$.

\#: significantly different from the DEX (ib, $62.5 \mu \mathrm{g}$ /ovary) group $(\mathrm{P}<0.05)$.

secretion to that of control groups (Fig. 2-A). Treatment from D4 raised plasma steroid levels compared to controls (significantly different on D5, 7, 9, 10) (Fig. 2-B). Treatment from D6 brought about more prominent effects (Fig. 2-C). The experimental groups exhibited higher progesterone levels on D6, 8, 10, 12 and 13, and vigorous progestational activity was prolonged about 5 days. A half dose (31.25 $\mu \mathrm{g}$ / ovary) also delayed the decline in plasma progesterone. In animals injected subcutaneously with IND from D6, plasma progesterone was instantly increased on D6 and D7 as compared to control or intrabursally injected groups (Fig. 2-D). This treatment showed significantly higher progesterone levels by D11 than controls, but the decrease to the basal level oc- curred earlier than locally injected groups.

\section{Effects of NDGA on progesterone secretion}

Similar to DEX or IND, local treatment with NDGA, a lipoxygenase inhibitor, from D2 made little difference in circulating progesterone levels (Fig. 3-A). Although its injection from D4 tended to increase the steroid level compared to control levels, a significant difference was observed only on D5 (Fig. 3-B). Treatment from D6 delayed the decrease in plasma progesterone to as late as D9, resulting in extension of the corpus luteum activity for only one day (Fig. 3-C). Doubling the dose of NDGA (125 $\mu \mathrm{g} /$ ovary) seemed to diminish such an effect. 

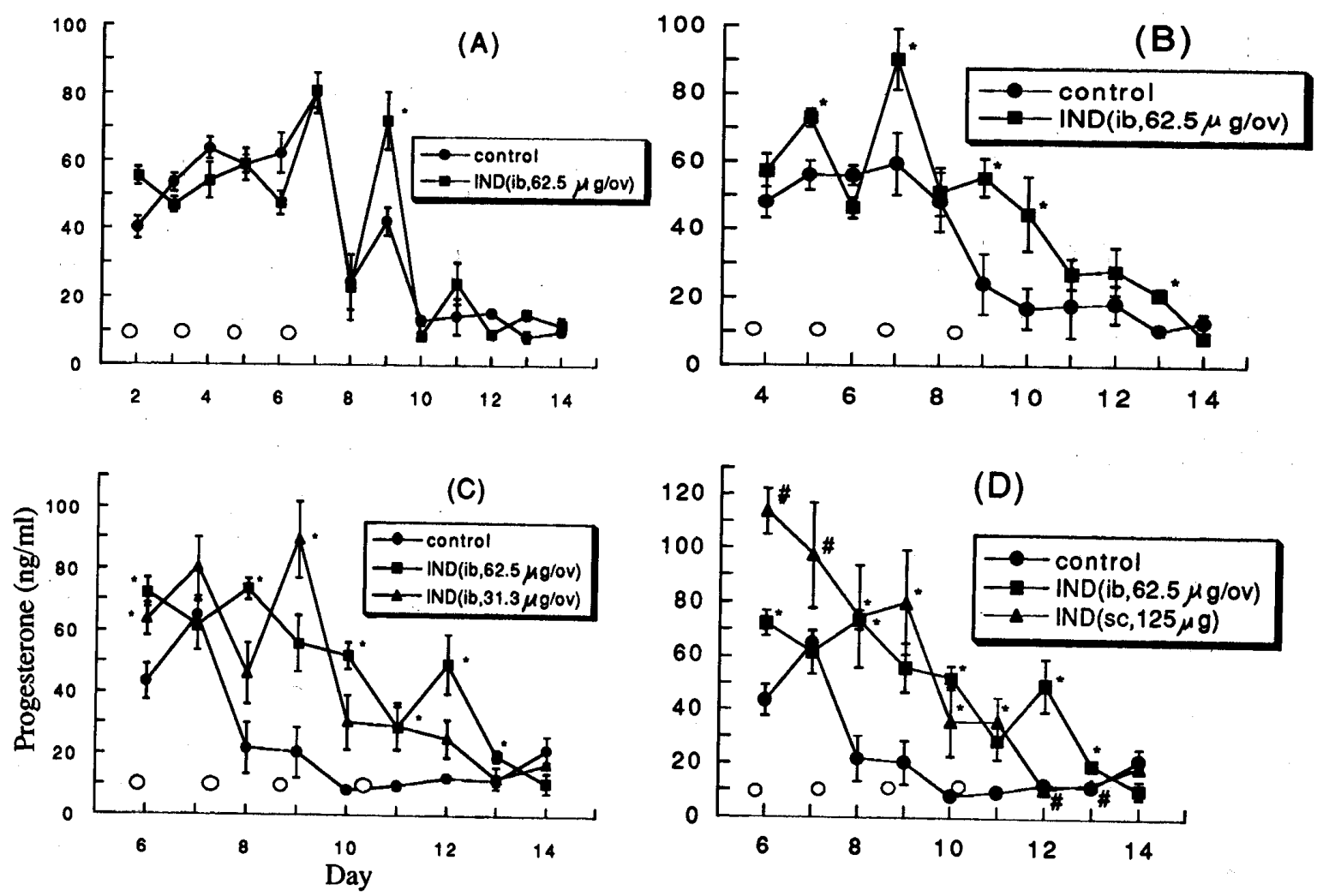

Fig. 2. Changes in plasma progesterone levels in immature pseudopregnant rats following intra-ovarian bursal (ib) or subcutaneous (sc) injections of IND. Details are the same as in Fig. 1. The doses of IND in a single injection were $62.5 \mu \mathrm{g} /$ ovary $(125 \mu \mathrm{g} / \mathrm{rat})$ and $31.25 \mu \mathrm{g} /$ ovary $(62.5 \mu \mathrm{g} / \mathrm{rat})$ in ib injected groups and $125 \mu \mathrm{g} /$ rat in sc injected groups.

*: significantly different from the control group $(\mathrm{P}<0.05)$.

\#: significantly different from the IND (ib, $62.5 \mu \mathrm{g}$ /ovary) group $(\mathrm{P}<0.05)$.

\section{Effects of IMZ on progesterone secretion}

The effect of administering IMZ (62.5 $\mu \mathrm{g} / \mathrm{ova}-$ ry), a supposed inhibitor of thromboxane synthase, from D6 was further examined (Fig. 4). Rats in the experimental group showed a similar change in extended progesterone secretion as compared to the animals receiving the preceeding inhibitors. Significant differences in the plasma steroid levels appeared on D8 and D10.

\section{Discussion}

The present study demonstrates that modulation of ovarian eicosanoid metabolism following local and repeated treatments with their synthesis inhibitors resulted in the delay of luteolysis in immature pseudopregnant rats. These results suggest that ovarian endogenous eicosanoids are involved in regulation of rat luteal activity and lifespan.

First, DEX challenge at the late luteal phase (from D6 to D10) delayed luteolysis in the present animal model but not from D2 or D4. DEX is known to suppress arachidonate production through inhibition of phospholipase $\mathrm{A}_{2}\left(\mathrm{PLA}_{2}\right)$ activity [12, 13]. In a similar pseudopregnant model, microsomal $\mathrm{PLA}_{2}$ activity, lipid peroxides level in the whole ovary $[14,15]$ and a $85 \mathrm{kDa}$ cytosolic PLA $\left.\mathrm{PLPA}_{2}\right)$ protein level in corpora lutea (Kurusu et al., unpublished data) increased as the plasma 

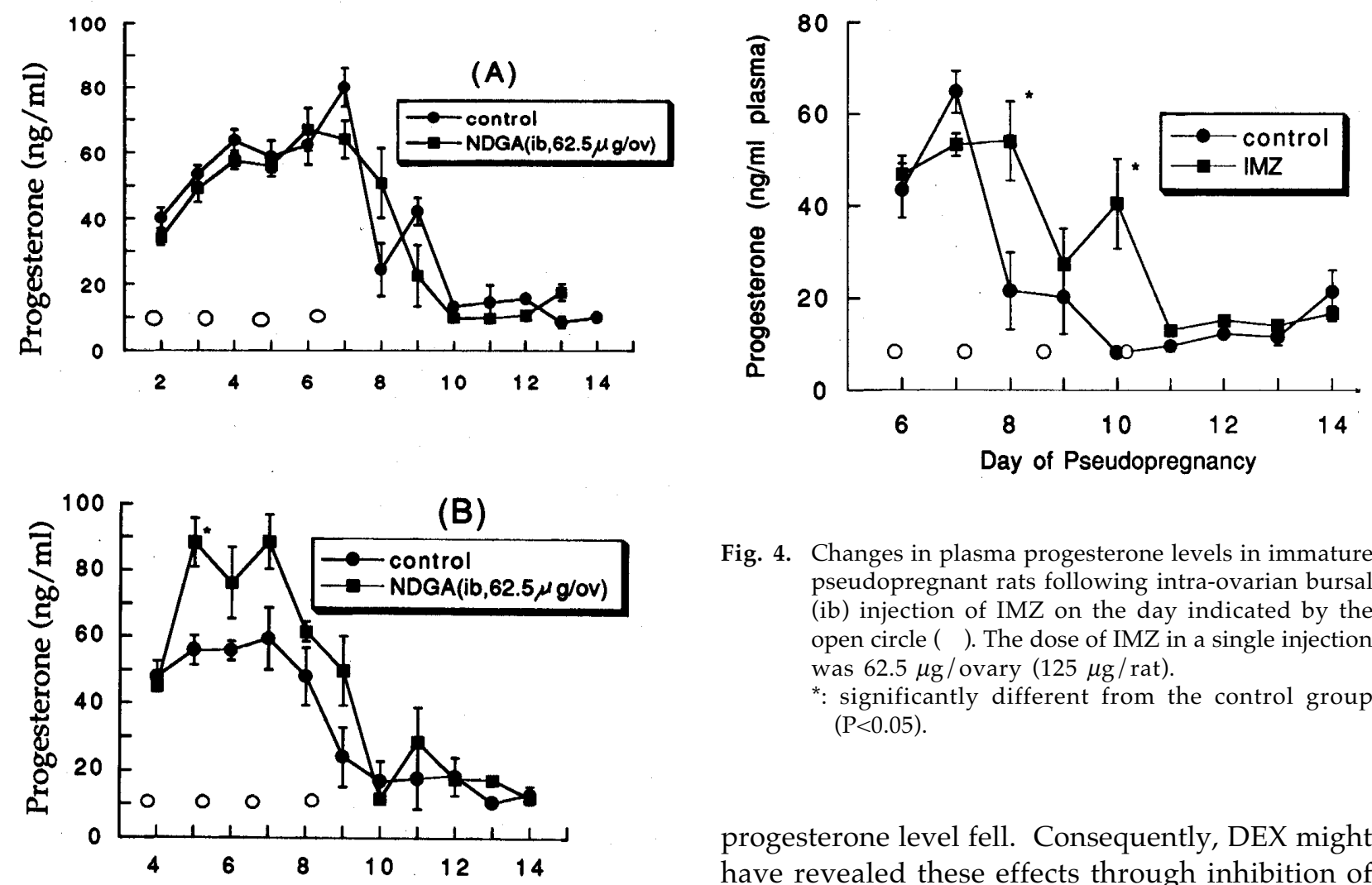

Fig. 4. Changes in plasma progesterone levels in immature pseudopregnant rats following intra-ovarian bursal (ib) injection of IMZ on the day indicated by the open circle $(\bigcirc)$. The dose of IMZ in a single injection was $62.5 \mu \mathrm{g} /$ ovary $(125 \mu \mathrm{g} / \mathrm{rat})$.

*: significantly different from the control group $(\mathrm{P}<0.05)$.

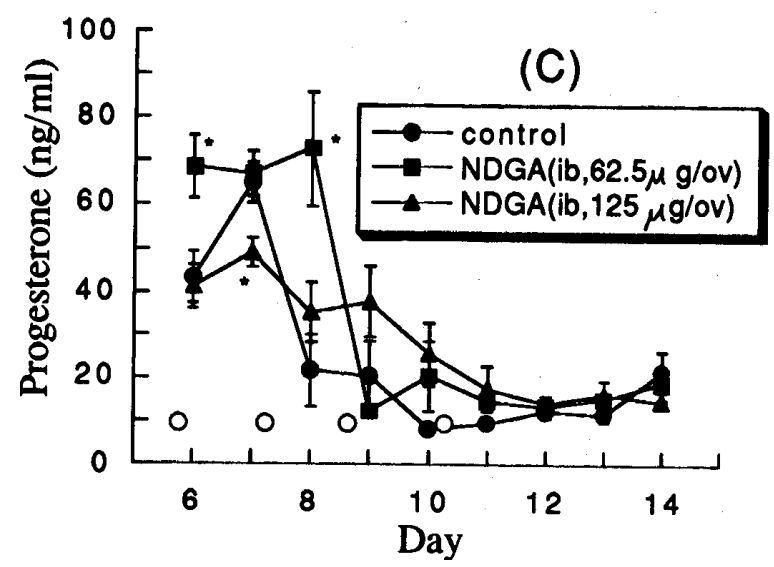

progesterone level fell. Consequently, DEX might have revealed these effects through inhibition of the increased $\mathrm{PLA}_{2}$ activity and subsequent eicosanoids production. This was further supported by the results of experiments using IND or NDGA.

Both cyclooxygenase (IND) and lipoxygenase inhibitors (NDGA) delayed luteal regression by treatment from D6, and IND was more potent in delaying luteolysis than NDGA at the dose of 62.5 $\mu \mathrm{g} /$ ovary $(125 \mu \mathrm{g} /$ rat $)$. Furthermore, the half dose of IND sustained the effect of extending active progestational activity, while the doubled dose of NDGA (125 $\mu \mathrm{g}$ / ovary) appeared to diminish the effect. In immature pseudopregnant rats without luteotropic stimulation by exogenous prolactin, luteal $\mathrm{PGF}_{2 \alpha}$ showed a temporal rise on D4-5 just before plasma progesterone began to fall on D6 [6]. In addition, luteal concentrations of $\mathrm{PGF}_{2 \alpha}$ $\mathrm{PGE}_{2}$ and 6-keto-PGF $\mathrm{PG}_{1 \alpha}$ (the stable metabolite of

Fig. 3. Changes in plasma progesterone levels in immature pseudopregnant rats following intra-ovarian bursal (ib) injections of NDGA. Details are the same as Fig. 1. The doses of NDGA in a single injection were $62.5 \mu \mathrm{g}$ / ovary $(125 \mu \mathrm{g} /$ rat $)$ or $125 \mu \mathrm{g} /$ ovary $(250$ $\mu \mathrm{g} / \mathrm{rat})$.

*: significantly different from the control group $(\mathrm{P}<0.05)$. $\mathrm{PGI}_{2}$ ) moderately increased or remained high during the luteal regression phase in adult pseudopregnant rats while declining in pregnant rats $[3,5]$. Taken together, the increase or persistence in prostanoid biosynthesis in the late luteal phase is thought to be an important process in initiation of luteolysis. While both $\mathrm{PGE}_{2}$ and $\mathrm{PGI}_{2}$ are supposed to be luteotropic in vitro [16], the 
overall effects of prostanoids during the late luteal phase would be luteolytic. The mechanism by which NDGA affects luteal activity seems to be complex. The reason for variations in the response to NDGA treatment is unclear. Because NDGA did not affect [17] or increased [18] arachidonate metabolites of the cyclooxygenase pathway, the high dose of NDGA, through inhibition of the lipoxygenase pathway, might induce a rise in the arachidonate pool available for prostanoid biosynthesis. Two kinds of eicosanoids of the lipoxygenase pathway are synthesized in human luteal cells and suppress its ability to secrete progesterone $[19,20]$. Whether eicosanoids of the lipoxygenase pathway are involved in regulation of rat luteal function is obscure at present. But their luteolytic potencies, even if they have any, may be less than those of cyclooxygenase metabolites. In the present study, the thromboxane synthase inhibitor IMZ also induced a sustained secretion of progesterone but was less potent than DEX or IND at the dose of $62.5 \mu \mathrm{g}$ / ovary as to the extent of the modulation of progesterone secretion. This suggests a possibility that luteal prostanoids, possibly other than thromboxanes, may be more potently involved in mechanisms of luteolysis.

In accordance with previous reports [7, 8], systemic administration of DEX or IND prolonged luteal activity in the present study. According to Espey et al. [10], systemic injection of IND $(0.1 \mathrm{mg} /$ immature rat ) decreased ovarian PGE and PGF contents to less than $10 \%$ in $5 \mathrm{~h}$. While it is described that a single dose of IND can reduce PG levels in the entire body for at least $24 \mathrm{~h} \mathrm{[21],} \mathrm{this}$ drug disappears rapidly from circulation with a plasma half-life of about $6 \mathrm{~h}[22,23]$. Furthermore, in rabbits injected intravenously with $10 \mathrm{mg} /$ $\mathrm{kg}$ IND (about 10 times higher than the present dose), ovarian prostaglandin production at 14-16 $\mathrm{h}$ after injection was similar to that of control rabbit ovaries [24]. Therefore, it is more likely that ovarian eicosanoids must have been suppressed in both locally animal and systemically administrated animals shortly after treatment, but the suppression must have persisted much longer in locally treated animals because of its high concentrations in the ovary. Moreover, the drugs gradually leaked out of the ovarian bursa in locally injected animals, and dilution in the systemic plasma would render them ineffective for the other tissues.

Uterine involvement in regulation of luteal function may be possible because the uterus produces luteolytic $\mathrm{PGF}_{2 \alpha}[1,5]$, and hysterectomy delays luteal regression in immature pseudopregnant rats [8]. However, Olofsson et al. showed that: 1) $\mathrm{PGF}_{2 \alpha}$ in luteal contents in vivo and produced by the isolated corpora lutea in vitro increased toward the end of the luteal phase in adult pseudopregnant rats, 2) the increase in luteal contents also persisted in hysterectomized rats, and 3) $\mathrm{PGF}_{2 \alpha}$ content in the remainder of the ovary was unaltered during the luteal phase [2-4]. Taken together with our present result that local treatment was more effective than systemic treatment in delaying luteolysis, it is suggested that the rat corpus luteum can intrinsically produce any amount of eicosanoids, which play an important role in the demise of luteal function. This appears to support the previous hypothesis of Rothchild [25]. The uterus may influence luteal eicosanoid production. It is noteworthy that exogenous cloprostenol $\left(\mathrm{PGF}_{2 \alpha}\right.$ analogue) increased the luteal synthesis of $\mathrm{PGF}_{2 \alpha}$ in vitro $[26,27]$.

Unexpectedly, systemic injections with DEX or IND induced an instant and significant increase in circulating progesterone. This suggests a possible mechanism in extra-ovarian tissue(s) which appear to negatively regulate luteal function through prostanoids production. The effect of systemically injected inhibitors on luteal activity may be mediated by substances other than eicosanoids. There are some reports that the secretion of LH or prolactin, which is relevant to rat luteal function, is modulated by arachidonate or eicosanoids in the rat pituitary [28-31].

In conclusion, modulations in ovarian endogenous eicosanoids by local administration with several kinds of synthesis inhibitors prolonged luteal activity in immature pseudopregnant rats. The present findings suggest that ovarian endogenous eicosanoids in the late luteal phase may be involved in initiation of functional luteolysis in rats. 


\section{References}

1. Poyser NL, Scott FM. Prostaglandin and thromboxane production by the rat uterus and ovary in vitro during the oestrous cycle. J Reprod Fert 1980; 60: 33-40.

2. Olofsson J, Norjavaara E, Selstam G. Synthesis of prostaglandin $\mathrm{F}_{2 \alpha}, \mathrm{E}_{2}$ and prostacyclin in isolated corpora lutea of adult pseudopregnant rats throughout the luteal life-span. PG LT Ess Fatty Acids 1992; 46: 151-161.

3. Olofsson J, Norjavaara E, Selstam G. In vivo levels of prostaglandin $\mathrm{F}_{2 \alpha}, \mathrm{E}_{2}$ and prostacyclin in the corpus luteum of pregnant and pseudopregnant rats. Biol Reprod 1990; 42: 792-800.

4. Olofsson J, Norjavaara E. Effects of hysterectomy and uterine deciduolization on in vivo levels of prostaglandins in the corpus luteum of adult pseudopregnant rats. Biol Reprod 1990; 43: 762768.

5. Cao L, Chan WY. Effects of oxytocin and uterine and luteal prostaglandins on the functional regression of the corpus luteum in pseudopregnant rats. J Reprod Fert 1993; 99: 181-186.

6. Kurusu S, Ohyama T, Watanabe H, Miyamoto $K$, Sumi K, Hisatsune $R$, Kawaminami $M$, Hashimoto I. Changes in plasma progestins levels and luteal prostaglandin $\mathrm{F}_{2 \alpha}$ content in immature pseudopregnant rats. J Reprod Dev 1994; 40: 85-89.

7. Lau IF, Saksena SK, Chang MC. Effect of indomethacin, an inhibitor of prostaglandin biosynthesis, on the length of pseudopregnancy in rats and hamsters. Acta Endocrinol 1975; 78: 343348.

8. Wang F, Riley JCM, Behrman HR. Immunosuppressive levels of glucocorticoid block extrauterine luteolysis in the rat. Biol Reprod 1993; 49: 66-73.

9. Sargent EL, Baughman WL, Novy MJ, Stouffer RL. Intraluteal infusion of a prostaglandin synthesis inhibitor, sodium meclofenamate, causes premature luteolysis in rhesus monkey. Endocrinology 1988; 123: 2261-2269.

10. Espey LL, Tanaka N, Okamura $\mathbf{H}$. Increase in ovarian leukotrienes during hormonally induced ovulation in the rat. Am J Physiol 1989; 256: E753759.

11. Hashimoto I, Asano T, Wiest WG. Progestational function of perfused rat corpora lutea. Endocrinology 1975; 96: 421-430.

12. Nakano T, Ohara O, Teraoka H, Arita H. Glucocorticoids suppress group 2 phospholipase $\mathrm{A}_{2}$ production by blocking mRNA synthesis and post transcriptional expression. J Biol Chem 1990; 265: 12745-12748.

13. Lin LL, Lin AY, DeWitt DL. Interleukin- $1 \alpha$ in- duces the accumulation of cytosolic phospholipase $\mathrm{A}_{2}$ and the release of prostaglandin $\mathrm{E}_{2}$ in human fibroblasts. J Biol Chem 1992; 267: 23451-23454.

14. Wu XM, Carlson JC. Alterations in phospholipase $\mathrm{A}_{2}$ activity during luteal regression in pseudopregnant and pregnant rats. Endocrinology 1990; 127: 2464-2468.

15. Kurusu S, Tsukamoto $\mathbf{K}$, Konishi $\mathbf{H}$, Tachibana M, Kawaminami M, Hashimoto I. Changes in ovarian lipid peroxides during pseudopregnancy and prostaglandin $\mathrm{F}_{2 \alpha}$ - induced luteolysis in the immature rats. In: Program of 9th International Congress of Endocrinology; 1992; Nice, France. Abstract 499.

16. Speroff L, Ramwell PW. Prostaglandin stimulation of in vitro progesterone synthesis. J Clin Endocrinol Metab 1970; 30: 345-350.

17. Salari H, Braquet $\mathbf{P}$, Borgeat $\mathbf{P}$. Comparative effects of indomethacin, acetylenic acids, 15-HETE, nordihydroguaiaretic acid and BW 755C on the metabolism of arachidonic acid in human leukocytes and platelets. PG LT Med 1984; 13: 53-59.

18. Milvae RA, Alila HW, Hansel W. Involvement of lipoxygenase products of arachidonic acid metabolism in bovine luteal function. Biol Reprod 1986; 35: 1210-1215.

19. Ichikawa F, Yoshimura Y, Oda T, Shiraki M, Maruyama K, Kawakami S, Nakamura $T$, Fukushima M. The effects of lipoxygenase products on progesterone and prostaglandin production by human corpora lutea. J Clin Endocrinol Metab 1990; 70: 849-855.

20. Yoshimura $\mathbf{Y}$, Nakamura $\mathbf{Y}$, Ichikawa F, Oda T, Jinno T, Ando M, Koyama N, Shiokawa N. Possible involvement of leukotriens in human luteal function. Acta Endocrinol 1992; 127: 246-251.

21. Flower RJ. Drugs which inhibit prostaglandin biosynthesis. Pharmacol Rev 1974; 26: 33-67.

22. Hucker HB, Zacchei AG, Cox SV, Brodie DA, Cantwell NHR. Studies on the absorption, distribution and excretion of indomethacin in various species. J Pharmcol Exp Ther 1966; 153: 237-239.

23. Domenech J, Lauroba J, MorenoJ, Pla-Delfina JM. A relationship between biological half-life and effective half-life according to pharmacological response in non-steroidal antiinflammatory drugs. Arzneim Forsch 1981; 31: 445-452.

24. Katz E, Dharmarajan AM, Sueoka K, Ghodgaonkar RB, Dubin NH, Wallach EE. Effects of systemic administration of indomethacin on ovulation, luteinization, and steroidogenesis in the rabbit ovary. Am J Obstet Gynecol 1989; 161: 1361-1366. 
25. Rothchild I. The regulation of the mammalian corpus luteum. Rec Prog Horm Res 1981; 37: 183298.

26. McDougall AN, Walker M, Watson J. The effect of cloprostenol on human luteal steroid and prostaglandin secretion in vitro. Br J Pharmacol 1977; 60: 425-431.

27. Waterman RA, Guthrie HD. Effects of cloprostenol administration on neutral lipid and prostaglandin F metabolism by porcine luteal tissue. Prostaglandins 1984; 27: 131-146.

28. Chobsieng $\mathbf{P}$, Naor Z, Koch $\mathbf{Y}$, Zor V, Lindner HR. Stimulatory effect of $\mathrm{PGE}_{2}$ on $\mathrm{LH}$ release in the rat: Evidence for hypothalamic site for action. Neuroendocrinology 1975; 17: 12-19.
29. Miyake A, Tasaka K, Mori S, Saito Y, Aono T. Prostaglandin $\mathrm{D}_{2}$ stimulates secretion of luteinizing hormone from pituitary gland in vitro. Acta Endocrinol 1983; 104: 164-170.

30. Canonico PL, Schettini G, Valdenegro CA, MacLeod RM. Arachidonic acid metabolism and prolactin secretion in vitro: a possible role for the lipoxygenase products. Neuroendocrinology 1983; 37: 212-216.

31. Canonico PL, Judd AM, Koike K, Valdenegro CA, MacLeod RM. Arachidonate stimulates prolactin release in vitro: a role for the fatty acid and its metabolites as intracellular regulator(s) in mammotrophs. Endocrinology 1985; 116: 218-224. 